\title{
¿Cómo Intervenir el Proceso de Caries en Adultos? Adaptación del Consenso de ORCA/EFCD/DGZ.
}

\section{How to Intervene the Caries Process in Adults? Adaptation of the ORCA/EFCD/DGZ Consensus.}

\author{
Constanza Echeverría ${ }^{1}$, Constanza E. Fernández ${ }^{1}$, Sebastián Valdés ${ }^{1}$, Ruth M. Santamaría ${ }^{2}$, \\ Christian Splieth ${ }^{2}$, Sebastian Paris ${ }^{3}$, Falk Schwendicke ${ }^{3}$, Rodrigo A. Giacaman ${ }^{1 *}$
}

\author{
1. Cariology Unit, Department of Oral Rehabilitation, \\ Faculty of Health Sciences, University of Talca, \\ Talca, Chile. \\ 2. Department of Preventive and Pediatric Dentistry, \\ University of Greifswald, Greifswald, Germany. \\ 3. Department of Operative Dentistry, Charité \\ Universitätsmedizin, Berlin, Berlin, Germany \\ * Correspondencia Autor: Rodrigo A. Giacaman | \\ Dirección: 1 Poniente 1141, Talca, Chile | Teléfono: \\ 56-71-2201546 | E-mail: giacaman@utalca.cl \\ Trabajo recibido el 02/12/2020. \\ Trabajo revisado 29/12/2020 \\ Aprobado para su publicación el 24/01/2021
}

\begin{abstract}
RESUMEN
El manejo terapéutico de lesiones de caries primarias y secundarias concentra gran parte del quehacer de los dentistas en el mundo. Recientes cambios en la concepción de la enfermedad de caries llevaron a un panel de expertos de la Organización Europea para la Investigación en Caries (ORCA), la Federación Europea de Odontología Conservadora (EFCD) y la Federación Alemana de Odontología Conservadora (DGZ) a analizar la evidencia y consensuar recomendaciones sobre manejo de caries en adultos. Mediante una reunión en Berlín, Alemania en 2019 y con metodología e-Delphi, los expertos analizaron la evidencia y propusieron recomendaciones clínicas. El propósito de este artículo es presentar una adaptación idiomática de las principales recomendaciones, que incluyen terapias no invasivas (higiene, uso de fluoruros y control de dieta), terapias microinvasiva (sellantes e infiltrantes), terapias necesariamente invasivas y la reparación de restauraciones. Todas las recomendaciones se basan en un enfoque mínimamente invasivo, con un adecuado manejo restaurador. Los dentistas de países hispanoparlantes podrán encontrar recomendaciones basadas en evidencia, provenientes de un consenso de expertos a nivel global, que orienten sus decisiones clínicas, apoyándose en los principios de la odontología de mínima intervención.
\end{abstract}

\section{PALABRAS CLAVE:}

Adultos; Caries dental; Tratamiento de caries; Flúor; Infiltrantes; Sellantes; Odontología de Mínima Intervención; Consenso ORCA-EFCD.

Int. J. Inter. Dent Vol. 14(1); 32-36, 2021.

\section{ABSTRACT}

Therapeutic management of primary and secondary caries lesions concentrates much of the work of dentists throughout the world. Recent changes in caries disease conception and therapeutic management led a panel of experts from the European Organisation for Caries Research (ORCA), the European Federation for Conservative Dentistry (EFCD) and the German Federation for Conservative Dentistry (DGZ) to analyze the evidence and reach consensus on recommendations for caries management in adults. Through a meeting held in Berlin, Germany in 2019 and using an e-Delphi methodology, the experts analyzed the evidence and proposed clinical recommendations. The purpose of this article is to present an idiomatic adaptation to Spanish of the main recommendations, which include non-invasive therapies (hygiene, use of fluoride and diet control), microinvasive therapies (sealants and infiltrants), invasive therapies and repair of restorations. All recommendations are based on a minimally invasive dentistry approach, with a technically adequate restorative management. Spanish-speaking dentists may use these consensus recommendations to guide their clinical decisions, based on the most recent evidence and experts opinions, under the principles of minimal intervention dentistry. KEY WORDS:

Adults; Dental caries; Caries treatment; Fluoride, Infiltrants; Sealants; Minimal Intervention Dentistry; Consensus ORCA-EFCD.

Int. J. Inter. Dent Vol. 14(1); 32-36, 2021. 


\section{INTRODUCCIÓN}

Los conceptos actuales sobre la enfermedad de caries y su consecuencia; las lesiones de caries, han variado sustancialmente en los últimos años ${ }^{(1)}$. La caries dental es actualmente entendida como una enfermedad crónica, no transmisible, causada por la exposición frecuente a carbohidratos fermentables de la dieta, modulada por factores biológicos, conductuales, ambientales y psicosociales ${ }^{(2)}$. Estas nuevas concepciones han hecho cambiar el foco de la odontología clínica desde una mirada predominantemente restauradora hacia el incorporar un mayor énfasis en la preservación de la estructura dental o "capital biológico"(3).

En general, las priorizaciones en salud bucal han recaído en la población infantil, dejando de lado a los adultos, quienes son igualmente afectados por la enfermedad. De hecho, los adultos tienen una alta prevalencia y severidad de caries tanto a nivel latinoamericano ${ }^{(4,5)}$, como en otras regiones del mundo ${ }^{(6,7)}$. En este grupo etario, las lesiones de caries proximales y de caries alrededor de restauraciones o secundarias, continúan siendo un problema que afecta a una parte importante de la población adulta. Una vez que un diente es restaurado, comienza un continuo proceso de recambio de restauraciones en el tiempo que termina mermando los remanentes biológicos e incluso causando la pérdida dentaria. Este fenómeno es conocido como "ciclo restaurador" o "espiral de la muerte"(8) y obliga a repensar el manejo de la enfermedad y de las lesiones de caries en pacientes adultos.

Los nuevos conceptos en Cariología clínica han tenido amplia difusión en distintos países, como por ejemplo en EE.UU.(9) y así también debe ocurrir en los países de la región Latinoamericana. Por lo anterior, este artículo es una adaptación de un reciente consenso de expertos a nivel global $^{(10)}$, convocado conjuntamente entre la Organización Europea para la Investigación en Caries (ORCA), la Federación Europea de Odontología Conservadora (EFCD) y la Federación Alemana de Odontología Conservadora (DGZ), quienes se reunieron en julio de 2019 en Berlín, Alemania para analizar la evidencia científica y consensuar recomendaciones clínicas. Previamente, estas organizaciones ya se habían reunido a consensuar orientaciones para los odontólogos clínicos sobre cuándo intervenir en el proceso de caries $^{(11)}$. Dado que el consenso de expertos se publicó originalmente en inglés ${ }^{(10)}$, los investigadores propusieron la adaptación idiomática para acercar las recomendaciones a diferentes regiones del mundo, en este caso, a países hispanos hablantes, con foco en Latinoamérica.

El consenso entrega recomendaciones para el manejo de lesiones de caries en adultos, específicamente en aquellas que ocurren en superficies proximales 0 asociadas a restauraciones previamente existentes, también denominadas caries secundarias ${ }^{(2)}$. Estos dos tipos de lesiones cariosas se incluyeron por ser las manifestaciones más comunes el proceso de caries en este grupo etario(12, 13). Así, el objetivo de este artículo es acercar las principales conclusiones alcanzadas en el consenso de ORCA/EFCD/DGZ ${ }^{(10)}$ para una mejor difusión y alcance a odontólogos hispanohablantes.

\section{Caries Dental en Población Adulta}

En general, ha habido un mayor énfasis en el abordaje de la caries en los rangos etarios extremos de la vida ${ }^{(14,15)}$, dejando al margen lo que ocurre en la adultez. Del mismo modo, en Latinoamérica hay escasos estudios que revisen esta temática en adultos.

El panel de expertos de ORCA/EFCD/DGZ concluye en el consenso que las lesiones de caries proximales y secundarias son el problema predominante y deberían ser el foco del manejo en la población adulta, argumentando las siguientes razones:

1) Las lesiones oclusales ocurren predominantemente en la infancia. Las lesiones de caries oclusales pueden ser exitosamente prevenidas a largo plazo mediante la utilización de sellantes de fosas y fisuras ${ }^{(16)}$. Debido a que los dientes recientemente erupcionados, principalmente los molares ${ }^{(14)}$, presentan mayor susceptibilidad a desarrollar lesiones cariosas tempranamente, su actividad genera una rápida progresión de la lesión. Así, las lesiones oclusales en dientes no sellados tienen una alta probabilidad de haber sido ya restauradas en la adolescencia (<18 años). Lo anterior conduce a una reducción en la incidencia y progresión de lesiones oclusales en la vida adulta ${ }^{(6)}$.

2) Escasa exposición de superficies radiculares. La exposición radicular determinará que lesiones cariosas pueden volverse más comunes en estas superficies ${ }^{(16)}$, dada la mayor susceptibilidad de la dentina comparada con el esmalte ${ }^{(17)}$. Sin embargo, las superficies radiculares expuestas no son frecuentemente evidentes en adultos ${ }^{(15)}$. Estas exposiciones radiculares ocurren tardíamente en el curso de vida, juntamente con una mayor pérdida de soporte periodontal, con o sin periodontitis asociada ${ }^{(18)}$.
3) Progresión lenta de lesiones proximales en dentición permanente. En pacientes con bajo riesgo o susceptibilidad a caries pueden pasar muchos años o incluso décadas para que ocurra alguna lesión proximal radiográficamente visible ${ }^{(6)}$. Por lo tanto, las lesiones proximales de dientes permanentes no se encuentran comúnmente en niños, debido que ha transcurrido poco tiempo para evidenciar su progresión. Además, la progresión lenta de caries proximales también se debe al uso masificado de fluoruros en el agua potable y dentífricos, siendo este último considerado la causa que explica la disminución mundial de la prevalencia de caries $^{(19,20)}$. Se ha descrito que en la cara mesial del primer molar permanente, en contacto con la superficie distal del segundo molar primario, se favorece la acumulación de un biofilm cariogénico, y probablemente la velocidad de progresión de la lesión en esa superficie (21). Las lesiones proximales pueden progresar incluso en poblaciones bien mantenidas y motivadas en países de ingresos altos ${ }^{(12)}$, por lo que el control periódico en el tiempo es fundamental en pacientes de bajo y alto riesgo de desarrollar lesiones de caries.

4) Existencia de restauraciones previas propicia la formación de lesiones secundarias. Las lesiones de caries secundaria, por definición, están asociadas a restauraciones existentes ${ }^{(2)}$. Aquellas restauraciones con márgenes a nivel proximal muestran mayor riesgo en comparación con aquellas limitadas o confinadas a la cara oclusal(22). No obstante, debido a la polarización de la caries dental(23) donde existe mayor número de población libre de lesiones que en el pasado, es posible que adultos jóvenes de hoy presenten menos restauraciones, lo que disminuye la probabilidad de desarrollar lesiones secundarias ${ }^{(7)}$.

\section{Importancia del Diagnóstico Precoz de Lesiones de Caries}

El avance de la lesión de caries es gradual y pasa por estadios iniciales de desmineralización del esmalte, para luego alcanzar la dentina ${ }^{(17,24)}$. El uso de criterios de diagnóstico como ICDAS (International Caries Detection and Assessment System), permite una diferenciación de las lesiones según diferentes estadios de severidad ${ }^{(25)}$. Este criterio permite un diagnóstico temprano y oportuno que sustentará el uso de estrategias de mínima intervención ${ }^{(26)}$. Asimismo, la actividad de la lesión es un criterio importante para determinar la necesidad de intervención. La cavitación, por otra parte, marca el punto de inflexión que conlleva a tener una conducta necesariamente invasiva ${ }^{(27)}$. El estado de cavitación debe ser juzgado objetivamente, utilizando métodos visuales o táctiles, además de la evaluación de la profundidad mediante radiografías complementarias ${ }^{(12)}$. Es importante recordar que la cavitación es poco probable en lesiones radiográficamente confinadas a esmalte (E1 y E2) o en lesiones que alcanzan el límite entre esmalte y dentina, o el tercio externo de la dentina (D1) (Figura 1) ${ }^{(6,12)}$. Por otra parte, la probabilidad de cavitación aumenta cuando se observa que la desmineralización ha alcanzado el tercio medio del espesor total de la dentina (D2). Las lesiones cavitadas por definición se consideran activas, excepto que estas sean autolimpiables donde el biofilm difícilmente puede acumularse ${ }^{(11)}$. Por ejemplo, dientes primarios anteriores con lesiones superficiales lisas abiertas o en lesiones superficiales radiculares pueden ser autolimpiables y mantenerse inactivas con medidas adecuadas no invasivas ${ }^{(11)}$. Como se analizará, el manejo de las lesiones de caries se debe centrar en controlar la enfermedad y no solo en limitar el daño generado. Consecuentemente, para tomar decisiones clínicas correctas es necesario integrar la evaluación del riesgo del paciente y de la lesión para optar por la mejor alternativa terapéutica.

\section{Intervención de Lesiones Proximales en el Adulto:}

La Figura 1 resume las recomendaciones para el manejo de lesiones de caries proximales en adultos, las cuales se dividen en:

a) Estrategias no invasivas (Figura 1a): no remueven tejido dental duro y se basan, por ejemplo, en la utilización de fluoruros tópicos y otros agentes químicos para el control del balance mineral del tejido dentario, medidas de control de biofilm y control de dieta ${ }^{(2,28,29)}$.

b) Estrategias microinvasivas (Figura 1b): remueven el tejido dental duro a nivel de micrones, usualmente durante el proceso de acondicionamiento con ácidos (grabado ácido), utilizado en técnicas de sellantes e infiltrantes ${ }^{(2,30,31)}$.

c) Estrategias operatorias necesariamente invasivas con filosofía de mínima intervención (Figura 1c): remueven una cantidad limitada de tejido dental duro, mediante el uso de excavadores manuales o instrumental rotatorio, proceso que es seguido por la colocación de un material restaurador ${ }^{(2,32)}$.

Todas las intervenciones, independientemente de si implican o no la 
remoción de tejidos duros, están bajo la filosofía de la Odontología de Mínima Intervención.

\section{a. Recomendaciones no invasivas:}

Existe amplio acuerdo en que las lesiones no cavitadas pueden ser detenidas en su avance utilizando técnicas que no implican el remover tejido duro del diente y por lo tanto estas deben ser manejadas no invasivamente, especialmente en pacientes de bajo riesgo o cuando las lesiones radiográficas están confinadas al esmalte (E1 y E2) (Figura 1a). Se deben evitar todas las estrategias restauradoras en lesiones no cavitadas.

Como se señaló, las estrategias no invasivas no remueven esmalte o dentina e incluyen la aplicación tópica de fluoruros, control mecánico del biofilm dental (cepillado y uso de seda), uso de dentífricos fluorurados, y el control de la dieta. La revisión sistemática con meta análisis ${ }^{(27)}$ que sirvió de insumo al panel de expertos del consenso original(10), destaca que la combinación de medidas de higiene oral y aplicación de fluoruros para el manejo de lesiones proximales es capaz de detener o reducir la progresión de lesiones de caries, pero calificó la evidencia como débil por el bajo número de estudios encontrados. Por su parte, la higiene oral por sí sola sin la utilización de fluoruros es capaz de remover placa y prevenir la gingivitis ${ }^{(33)}$, pero no necesariamente puede controlar las lesiones de caries existentes ${ }^{(34-36)}$. Por lo tanto, se recomienda la utilización de cepillado con dentífrico fluorado de más de 1.000 ppm $\mathrm{F}^{(34-36)}$. Para el uso de terapias tópicas profesionales, como el barniz o los geles de fluoruro, la evidencia también es débil puesto que no existen estudios que solamente utilicen este tipo de terapias. Sin embargo, existe evidencia para el uso tópico de fluoruros profesionales (ej. barniz) para el manejo de lesiones de caries en general, incluyendo niños y adolescentes ${ }^{(37,38)}$, y en diferentes superficies y denticiones ${ }^{(39,40)}$ En relación a la dieta, la fuerza de la evidencia se consideró como débil puesto que no existe datos específicos que demuestren que un control de dieta permite controlar las lesiones proximales. No obstante, existe una alta plausibilidad biológica y posibilidad de éxito ante la implementación de esta medida ${ }^{(41,42)}$. Se recomienda realizar un conjunto de medidas para evitar el desarrollo de lesiones de caries (Figura 1c). El uso de medidas no invasivas busca, por tanto, modificar el riesgo de caries del paciente, como parte fundamental en el control de la enfermedad. Es así como el riesgo individual de cada paciente es pieza clave cuando el clínico se ve enfrentado a la toma de decisiones de tratamiento. En la mayoría de las poblaciones con bajo riesgo de caries, la progresión de las lesiones a nivel de esmalte (E1 y E2) es lenta, tomando incluso varios años, mientras que la progresión es notablemente más rápida en lesiones cuya extensión ya involucra dentina (D1, D2 y D3). Por su parte, individuos con un alto riesgo de caries reportan una progresión notablemente más rápida, en donde el avance de una lesión no cavitada a una lesión cavitada en dentina puede tardar un período de 1 a 2 años. Generalmente, el éxito de las medidas no invasivas dependerá de la adherencia y del cambio de hábitos y conductas por parte de los pacientes. Entonces, el manejar el riesgo o susceptibilidad del paciente permitirá detener la progresión de lesiones cariosas, pero también prevendrá la formación de nuevas lesiones en aquellas superficies que no hayan sido afectadas previamente (Figura 1c).

\section{b. Recomendaciones Microinvasivas:}

En pacientes de alto riesgo o cuando las imágenes radiográficas se extienden en dentina es recomendable utilizar técnicas micro invasivas como sellantes e infiltrantes (Figura 1,b). El manejo microinvasivo de lesiones proximales cuenta con evidencia, con un número importante de ensayos clínicos randomizados, en donde se hacen comparaciones de los tratamientos microinvasivos combinados con medidas no invasivas contra solo realizar manejo no invasivo(2,28,43-45). Los estudios encontraron que los tratamientos microinvasivos combinados con medidas no invasivas detienen lesiones no cavitadas de esmalte y lesiones iniciales de dentina (limitadas al tercio externo de dentina radiográficamente (D1) y clínicamente no cavitadas), abordaje que es significativamente más efectivo que utilizar únicamente un manejo no invasivo ${ }^{(27)}$. Sin embargo, hay poca claridad sobre qué técnica ofrece mayor beneficio. Existe evidencia que avala que tanto sellantes como resinas infiltrantes pueden detener las lesiones limitadas al esmalte (E1 y E2) y alrededor de la unión esmalte-dentina (E2-D1), mientras que el uso de infiltrantes puede ser efectivo para lesiones que involucran dentina (D1) ${ }^{(30,31)}$ (Figura 1b). La decisión entre sellar o infiltrar debe ser guiada por consideraciones individuales, aplicabilidad, experiencia clínica y factibilidad financiera ${ }^{(10)}$.

\section{c. Recomendaciones Necesariamente Invasivas:}

Para el manejo mínimamente invasivo restaurador de lesiones proximales existe mayor número de estudios disponibles ${ }^{(46)}$. Cuando se restringe solo a restauraciones proximales, la resina compuesta muestra un riesgo de falla significativamente mayor que la amalgama ${ }^{(47)}$, en tanto, que existe ausencia de diferencias significativas entre otros materiales ${ }^{(47)}$. Dado que los estudios utilizan diferentes materiales y operadores, las comparaciones son difíciles, por lo que el panel calificó la evidencia como débil para este parámetro (Figura 1d).

Si bien la amalgama ha demostrado tener un menor riesgo de falla en cavidades proximales, las restauraciones adhesivas del color del diente son de primera elección entre pacientes y profesionales por el valor estético que proveen. A pesar de que la amalgama tiene una técnica menos sensible al operador y de menor riesgo de caries secundaria a nivel marginal( ${ }^{(47)}$, su utilización depende de las guías existentes en cada país. Si bien la amalgama es preferible para muchos escenarios complejos (ej. Dificultad para el aislamiento del campo operatorio), la actual reducción global en el uso de la amalgama dental pactada en el acuerdo de Minamata constituye una razón argumentada con frecuencia a la hora de no elegir la amalgama como material de elección ${ }^{(48)}$. Los materiales adhesivos, por su parte, permiten un abordaje mínimamente invasivo pues la adhesión química permite preparar el diente preservando estructura dentaria. Sin embargo, una correcta técnica y manipulación de materiales resinosos puede aumentar la longevidad de las restauraciones estéticas. Para defectos extensos, especialmente en dientes tratados endodónticamente, se ha considerado también la realización de restauraciones indirectas según sea la necesidad individual del caso (Figura 1d).

\section{Intervención de Lesiones Secundarias en el Adulto (Figura 2):}

La prevalencia de lesiones secundarias o en relación al margen de restauraciones existentes aumenta en pacientes con alto riesgo de caries $^{(13,49)}$, en el margen gingival de las restauraciones ${ }^{(50,51)}$ y en dientes posteriores ${ }^{(52)}$. Las lesiones cariosas asociadas a márgenes de restauraciones existentes pueden ser resultado de la enfermedad de caries no abordada o abordada con medidas insuficientes (relacionado al paciente)(22,53). Igualmente, estas lesiones pueden ser causadas por defectos marginales importantes como excesos o gaps en los márgenes de restauraciones (relacionado al operador). Las brechas o defectos marginales pueden ser el resultado de un emplazado inicial imperfecto de la restauración o deficiente manejo del tejido dentario(54). Se ha argumentado que estas fallas también puede ser el resultado de la degradación hidrolítica de la capa hibrida adhesiva de la restauración ${ }^{(55,56)}$. La existencia de brechas es perjudicial y aumentará el riesgo de caries, pues creará un área favorable para la retención de biofilm dental. Es

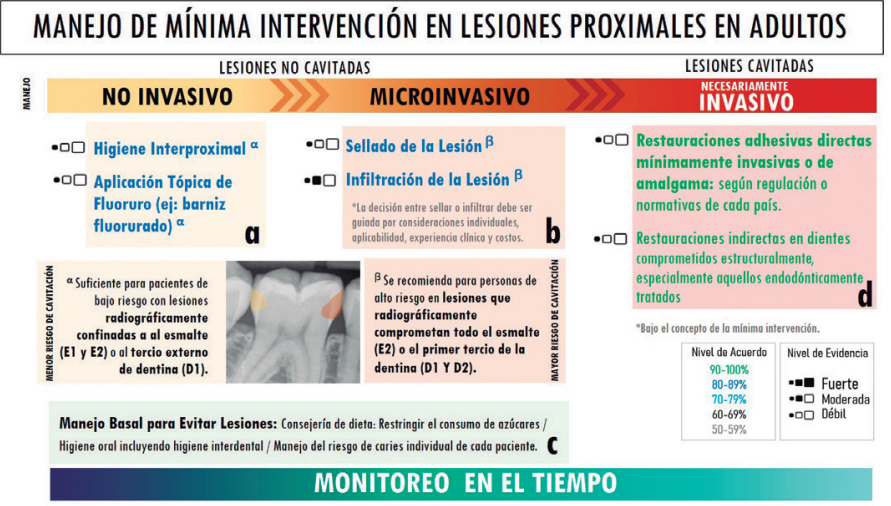

Figura 1.

\section{MANEJO DE MÍNIMA INTERVENCIÓN EN LESIONES ADYACENTES A RESTAURACIONES \\ RESTAURACIÓN SIN NECESIDAD DE REPARACIÓN RESTAURACIÓN DEFECTUOSA LESIONES CAVITADAS 量 \\ NO INVASIVO

MICROINVASIVO \\ NECESARIAMENTE}

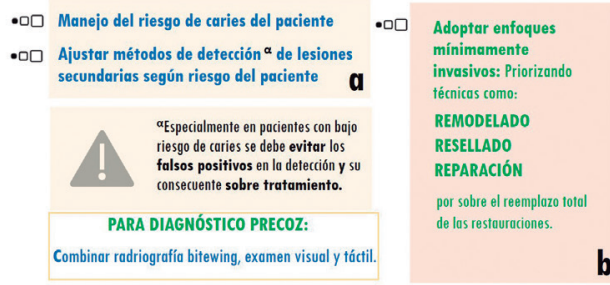

Adoptar enfoques mínimamente invasivos: teiido duro y vitalidad pulpor. C

MONITOREO EN EL TIEMPO

34 | Int. J. Inter. Dent Vol. 14(1); 32-36, 2021.

Figura 2. 
sabido que las áreas de acúmulo de biofilm no autolimpiables tenderán a ser activas y consecuentemente, conducirán a pérdida mineral y progresión de la lesión de caries. Una revisión sistemática previa sobre lesiones de caries secundarias ${ }^{(57)}$ no arrojó diferencias evidentes cuando se compararon distintos tipos de biomateriales dentales y de estrategias adhesivas ${ }^{(57)}$. Esta evidencia, por tanto, le resta importancia a la naturaleza y características del material per se, siempre y cuando la técnica de trabajo sea la correcta ${ }^{(57)}$. Consecuentemente, los factores relacionados con el paciente y con el operador son decisivos para controlar las lesiones asociadas a restauraciones, en tanto que las estrategias adhesivas y los materiales restauradores juegan un rol mucho menos relevante ${ }^{(10,27)}$.

Como fue previamente señalado, las restauraciones de amalgama se asocian a un menor riesgo de caries secundaria ${ }^{(47)}$, y de falla general en la mayoría de las cavidades. No existen diferencias relevantes en las tasas de éxito clínico en el tiempo entre los materiales estéticos, incluidos resinas compuestas, cementos de vidrio ionómero (VI), cementos de $\mathrm{VI}$ modificados con resina (VIMR) y resinas modificadas con poliácidos (compómeros) ${ }^{(27)}$. A pesar de que parece no importar el material, esta conclusión se debe tomar con cautela puesto que es condicionada por algunas limitaciones en la investigación en el área, por ejemplo, cortos periodos de seguimiento, tamaños muestrales limitados y bajo riesgo de caries individual de los participantes de los ensayos clínicos. Sin embargo, lo más importante es el concepto de restaurar solo cuando sea absolutamente necesario, restaurar con un enfoque de mínima intervención y manejar la enfermedad subyacente con estrategias no invasivas (Figuras 1a y 2a).

La detección temprana de lesiones secundarias puede permitir la aplicación de opciones de tratamientos menos invasivos como remodelación (tallado y pulido) de la superficie, resellado o reparación (Figura 2b), en lugar de la remoción completa de la restauración y su reemplazo (Figura 2c), permitiendo la preservación de estructura del diente y reduciendo el riesgo subsecuente de complicaciones de tratamiento ${ }^{(46)}$. Ante la sospecha de lesiones secundarias, estas deben ser manejas no invasivamente (Fig 2a) y monitoreadas regularmente. Para la detección temprana de lesiones de caries secundarias existe una variedad de métodos incluyendo evaluaciones visuales, táctiles, radiográficas, fluorescencia laser y fluorescencia inducida por luz cuantitativa $(\mathrm{QLF})^{(58)}$. Una reciente revisión sistemática concluyó que la detección visual, radiográfica y de fluorescencia láser, tienen similar habilidad de detectar superficies cariosas (sensibilidad) y sanas (especificidad) ${ }^{(58)}$. Por otro lado, los sistemas táctiles y de fluorescencia inducida por luz no son tan útiles cuando se utilizan de forma aislada. En general, es recomendable combinar herramientas que permitan la detección de lesiones clínicamente inaccesibles, como las radiografías de aleta mordida, conjuntamente con manejo visual y táctil, lo que facilitará la confirmación de hallazgos visibles (Figura 2a).

El manejo de las lesiones próximas a restauraciones o lesiones secundarias también debe ser bajo un enfoque de mínima intervención (Figura 2). Dado que gran porcentaje del tiempo clínico es destinado a recambiar restauraciones, existe una posibilidad cierta de sobre tratar, especialmente en pacientes de bajo riesgo de caries. Dado la lenta progresión de lesiones, ante la duda es recomendable revaluar en controles futuros $^{(59)}$. Por otro lado, a pesar de las ventajas obvias que tiene el enfoque mínimamente invasivo como reducción de tejido dental removido, disminución del daño pulpar, y prolongación de la vida útil del complejo diente-restauración, se requiere una cuidadosa selección de los casos. Debe considerarse que cuando no se hace una correcta selección de caso, pudiese tener un mayor costo en el largo plazo ${ }^{(10)}$ Para decidir el manejo de lesiones de caries próximas a restauraciones se debe considerar la situación clínica específica y preguntarse si es posible la reparación. Se deben además combinar los deseos del paciente, la experiencia y experticia del odontólogo con distintos tipos de tratamiento(10)

\section{CONCLUSIONES}

Un gran reto en el abordaje de caries en la población adulta es cómo evitar gatillar el ciclo de restauraciones con una aproximación excesivamente restauradora, la que finalmente compromete la longevidad del diente y los tejidos orales. Esto se hace especialmente relevante en momentos en que el mundo vive un envejecimiento acelerado de la población, con expectativas de vida mucho mayores.

Los pacientes adultos requieren de un manejo de la enfermedad de caries y de las lesiones de caries con un enfoque de mínima intervención, tanto con el uso de medidas no invasivas, microinvasivas y mínimamente invasivas. Las decisiones terapéuticas, además, deben tener una mirada personalizada, dependiendo de la situación local del diente y de los factores biológicos y sociales de cada persona, así como la experiencia del dentista.

Este consenso ofrece lineamientos generales, basados en evidencia para guiar al clínico con las recomendaciones originadas por expertos a nivel global en cómo abordar el proceso de caries en adultos.

\section{CONFLICTO DE INTERÉS}

Los autores no declaran conflicto de interés.

\section{FINANCIAMIENTO}

Este artículo fue financiado por recursos internos del Departamento de Rehabilitación Buco Máxilofacial de la Universidad de Talca, Chile.

\section{Bibliografía}

1. Innes NPT, Chu CH, Fontana M, Lo ECM, Thomson WM, Uribe S, et al. A Century of change towards prevention and minimal intervention in cariology. J Dent Res. 2019;98(6):611-7.

2. Machiulskiene V, Campus G, Carvalho JC, Dige I, Ekstrand KR, Jablonski-Momen $\mathrm{A}$, et al. Terminology of dental caries and dental caries management: consensus report of a workshop organized by ORCA and Cariology Research Group of IADR. Caries Res. 2020;54(1):7-14.

3. Giacaman R. Preserving healthy teeth throughout the life cycle, the biological asset. J Oral Res. 2019;6(4):80-1.

4. García-Cortés JO, Medina-Solís CE, Loyola-Rodriguez JP, Mejía-Cruz JA Medina-Cerda E, Patiño-Marín N, et al. Dental caries' experience, prevalence and severity in Mexican adolescents and young adults. Rev Salud Publica (Bogota). 2009;11(1):82-91.

5. Soares GH, Pereira NF, Biazevic MGH, Braga MM, Michel-Crosato E. Dental caries in South American Indigenous peoples: A systematic review. Community Dent Oral Epidemiol. 2019;47(2):142-52.

6. Mejàre I, Stenlund $\mathrm{H}$, Zelezny-Holmlund $\mathrm{C}$. Caries incidence and lesion progression from adolescence to young adulthood: a prospective 15-year cohort study in Sweden. Caries Res. 2004;38(2):130-41

7. Jordan RA, Krois J, Schiffner U, Micheelis W, Schwendicke F. Trends in caries experience in the permanent dentition in Germany 1997-2014, and projection to 2030: Morbidity shifts in an aging society. Sci Rep. 2019;9(1):5534.

8. Elderton RJ. Clinical studies concerning re-restoration of teeth. Adv Dent Res. 1990;4:4-9.

9. Fernandez CE, Gonzalez-Cabezas C, Fontana M. Minimum intervention dentistry in the US: an update from a cariology perspective. Br Dent J. 2020;229(7):483-6.

10. Schwendicke F, Splieth CH, Bottenberg P, Breschi L, Campus G, Doméjean S, et al. How to intervene in the caries process in adults: proximal and secondary caries? An EFCD-ORCA-DGZ expert Delphi consensus statement. Clin Oral Investig. 2020;24(9):3315-21.

11. Schwendicke F, Splieth C, Breschi L, Banerjee A, Fontana M, Paris S, et al When to intervene in the caries process? An expert Delphi consensus statement.
Clin Oral Investig. 2019;23(10):3691-703

12. Mejare I, Kallest I C, Stenlund $H$. Incidence and progression of approximal caries from 11 to 22 years of age in Sweden: A prospective radiographic study. Caries Res. 1999:33(2):93-100.

13. van de Sande FH, Opdam NJ, Rodolpho PA, Correa MB, Demarco FF, Cenci MS. Patient risk factors' influence on survival of posterior composites. J Dent Res. 2013;92(7 Suppl):78S-83S.

14. Broadbent JM, Foster Page LA, Thomson WM, Poulton R. Permanent dentition caries through the first half of life. Br Dent J. 2013;215(7):E12.

15. Broadbent JM, Thomson WM, Poulton R. Trajectory patterns of dental caries experience in the permanent dentition to the fourth decade of life. J Dent Res. 2008;87(1):69-72

16. Ahovuo-Saloranta A, Forss H, Walsh T, Nordblad A, Makela M, Worthington HV. Pit and fissure sealants for preventing dental decay in permanent teeth. Cochrane Database Syst Rev. 2017;7:CD001830.

17. Fejerskov $O$. Concepts of dental caries and their consequences for understanding the disease. Community Dent Oral Epidemiol. 1997;25(1):5-12.

18. Papapanou PN, Sanz M, Buduneli N, Dietrich T, Feres M, Fine DH, et al. Periodontitis: Consensus report of workgroup 2 of the 2017 World Workshop on the Classification of Periodontal and Peri-Implant Diseases and Conditions. J Periodontol. 2018;89 Suppl 1:S173-S82.

19. Bratthall D, Hansel-Petersson G, Sundberg $H$. Reasons for the caries decline: what do the experts believe? Eur J Oral Sci. 1996;104(4 ( Pt 2)):416-22; discussion 23-25, 30-32.

20. Cury JA, Tenuta LM, Ribeiro CC, Paes Leme AF. The importance of fluoride dentifrices to the current dental caries prevalence in Brazil. Braz Dent $\mathrm{J}$. 2004;15(3):167-74

21. Mejàre $I$, Stenlund $H$, Julihn $A$, Larsson I, Permert L. Influence of approximal caries in primary molars on caries rate for the mesial surface of the first permanent molar in swedish children from 6 to 12 years of age. Caries Res. 2001;35(3):178-85. 22. Ferracane JL. Models of caries formation around dental composite restorations. J Dent Res. 2017:96(4):364-71. 
23. Tanner T, Kämppi A, Päkkilä J, Patinen P, Rosberg J, Karjalainen K, et al. Prevalence and polarization of dental caries among young, healthy adults: Crosssectional epidemiological study. Acta Odontol Scand. 2013;71(6):1436-42.

24. Pitts NB, Zero DT, Marsh PD, Ekstrand K, Weintraub JA, Ramos-Gomez F, et al. Dental caries. Nat Rev Dis Primers. 2017;3:17030.

25. Pitts NB, Ekstrand KR, Foundation ICDAS. International Caries Detection and Assessment System (ICDAS) and its International Caries Classification and Management System (ICCMS) - methods for staging of the caries process and enabling dentists to manage caries. Community Dent Oral Epidemiol. 2013;41(1):e41-52

26. Dennison JB, Hamilton JC. Treatment decisions and conservation of tooth structure. Dent Clin North Am. 2005;49(4):825-45, vii.

27. Splieth $\mathrm{CH}$, Kanzow P, Wiegand A, Schmoeckel J, Jablonski-Momeni A. How to intervene in the caries process: proximal caries in adolescents and adults-a systematic review and meta-analysis. Clin Oral Investig. 2020;24(5):1623-36.

28. Bjørndal L. Buonocore Memorial Lecture. Dentin caries: progression and clinical management. Oper Dent. 2002;27(3):211-7.

29. Holmgren C, Gaucher C, Decerle N, Doméjean S. Minimal intervention dentistry II: part 3. Management of non-cavitated (initial) occlusal caries lesions--noninvasive approaches through remineralisation and therapeutic sealants. Br Dent $\mathrm{J}$. 2014;216(5):237-43.

30. Paris S, Meyer-Lueckel H, Cölfen H, Kielbassa AM. Resin infiltration of artificial enamel caries lesions with experimental light curing resins. Dent Mater J. 2007;26(4):582-8.

31. Martignon S, Ekstrand KR, Ellwood R. Efficacy of sealing proximal early active lesions: an 18-month clinical study evaluated by conventional and subtraction radiography. Caries Res. 2006;40(5):382-8.

32. Frencken JE, Peters MC, Manton DJ, Leal SC, Gordan VV, Eden E. Minimal intervention dentistry for managing dental caries - a review: report of a FDI task group. Int Dent J. 2012;62(5):223-43.

33. Wilder RS, Bray KS. Improving periodontal outcomes: merging clinical and behavioral science. Periodontol 2000. 2016;71(1):65-81.

34. Marinho VC, Higgins JP, Sheiham A, Logan S. Fluoride toothpastes for preventing dental caries in children and adolescents. Cochrane Database Syst Rev. 2003(1):CD002278.

35. Walsh T, Worthington HV, Glenny AM, Appelbe P, Marinho VC, Shi X. Fluoride toothpastes of different concentrations for preventing dental caries in children and adolescents. Cochrane Database Syst Rev. 2010(1):CD007868.

36. Walsh T, Worthington HV, Glenny AM, Marinho VC, Jeroncic A. Fluoride toothpastes of different concentrations for preventing dental caries. Cochrane Database Syst Rev. 2019;3:CD007868.

37. Marinho VC. Evidence-based effectiveness of topical fluorides. Adv Dent Res. 2008;20(1):3-7.

38. Marinho VC, Worthington HV, Walsh T, Clarkson JE. Fluoride varnishes for preventing dental caries in children and adolescents. Cochrane Database Syst Rev. 2013;7:CD002279.

39. Slayton RL, Urquhart O, Araujo MWB, Fontana M, Guzmán-Armstrong S Nascimento MM, et al. Evidence-based clinical practice guideline on nonrestorative treatments for carious lesions: A report from the American Dental Association. J Am Dent Assoc. 2018;149(10):837-49.e19.

40. Urquhart O, Tampi MP, Pilcher L, Slayton RL, Araujo MWB, Fontana M, et al.
Nonrestorative treatments for caries: systematic review and network meta-analysis. J Dent Res. 2019;98(1):14-26.

41. Chapple IL, Bouchard P, Cagetti MG, Campus G, Carra MC, Cocco F, et al. Interaction of lifestyle, behaviour or systemic diseases with dental caries and periodontal diseases: consensus report of group 2 of the joint EFP/ORCA workshop on the boundaries between caries and periodontal diseases. J Clin Periodontol. 2017;44 Suppl 18:S39-S51.

42. Hashem KM, He FJ, MacGregor GA. Effects of product reformulation on sugar intake and health-a systematic review and meta-analysis. Nutr Rev. 2019;77(3):18196.

43. Liang Y, Deng Z, Dai X, Tian J, Zhao W. Micro-invasive interventions for managing non-cavitated proximal caries of different depths: a systematic review and meta-analysis. Clin Oral Investig. 2018;22(8):2675-84.

44. Ammari MM, Soviero VM, da Silva Fidalgo TK, Lenzi M, Ferreira DM, Mattos CT, et al. Is non-cavitated proximal lesion sealing an effective method for caries contro in primary and permanent teeth? A systematic review and meta-analysis. J Dent. 2014;42(10):1217-27.

45. Yazıcıoğlu $\mathrm{O}$, Ulukapı $\mathrm{H}$. The investigation of non-invasive techniques for treating early approximal carious lesions: an in vivo study. Int Dent J. 2014;64(1):1-11.

46. Green D, Mackenzie L, Banerjee A. Minimally Invasive long-term management of direct restorations: the ' 5 Rs'. Dent Update. 2015;42(5):413-6, 9-21, 23-6.

47. Moraschini V, Fai CK, Alto RM, Dos Santos GO. Amalgam and resin composite longevity of posterior restorations: A systematic review and meta-analysis. J Dent. 2015;43(9):1043-50.

48. Program UNE. Minamata Convention on Mercury. United Nations, New York, 2013.

49. Opdam NJ, Bronkhorst EM, Roeters JM, Loomans BA. A retrospective clinical study on longevity of posterior composite and amalgam restorations. Dent Mater. 2007;23(1):2-8.

50. Mjor IA. Frequency of secondary caries at various anatomical locations. Oper Dent. 1985;10(3):88-92.

51. Mjör IA. Clinical diagnosis of recurrent caries. J Am Dent Assoc. 2005;136(10):1426-33

52. Demarco FF, Collares K, Coelho-de-Souza FH, Correa MB, Cenci MS, Moraes $\mathrm{RR}$, et al. Anterior composite restorations: A systematic review on long-term survival and reasons for failure. Dent Mater. 2015;31(10):1214-24.

53. Kidd EA. Diagnosis of secondary caries. J Dent Educ. 2001;65(10):997-1000

54. Vandewalle KS, Ferracane JL, Hilton TJ, Erickson RL, Sakaguchi RL. Effect of energy density on properties and marginal integrity of posterior resin composite restorations. Dent Mater. 2004;20(1):96-106.

55. Liu Y, Tjäderhane L, Breschi L, Mazzoni A, Li N, Mao J, et al. Limitations in bonding to dentin and experimental strategies to prevent bond degradation. J Dent Res. 2011;90(8):953-68.

56. Tjäderhane L. Dentin bonding: can we make it last? Oper Dent. 2015;40(1):4-18 57. Askar H, Krois J, Göstemeyer G, Bottenberg P, Zero D, Banerjee A, et al. Secondary caries: what is it, and how it can be controlled, detected, and managed? Clin Oral Investig. 2020;24(5):1869-76.

58. Brouwer F, Askar H, Paris S, Schwendicke F. Detecting secondary caries lesions: a systematic review and meta-analysis. J Dent Res. 2016;95(2):143-51.

59. Schwendicke F, Brouwer F, Paris S, Stolpe M. detecting proximal secondary caries lesions: a cost-effectiveness analysis. J Dent Res. 2016;95(2):152-9. 